\title{
Erratum zu: Leitlinie zum Management IgE-vermittelter Nahrungsmittelallergien
}

Erratum zu: Allergo J Int 2015;24:256-93

DOI: $10.1007 / \mathrm{s} 40629-015-0074-0$

In der Originalpublikation der Leitlinie ist in Tabelle 8 in der Zeile „Apfel“ das Allergen Mal d 1 beim Setzen verlorengegangen, wodurch auch die weiteren Apfelallergene den falschen Proteinfamilien zugeordnet wurden. In Tabelle 8 und Tabelle 9 wurde die Kennzeichnung einiger Allergene als „verfügbar für die In-vitro-Diagnostik“ ergänzt, da sie mittlerweile verfügbar sind. Die korrigierten und akutalisierten Tabellen finden Sie unten.

Darüber hinaus war in der Originalpublikation die DOI-Nummer in der Zitation, die auf die englischsprachige Version verweist, falsch. Die richtige DOI lautet 10.1007/s40629-015-0074-0. In der Zitation hat zudem leider der Autor Alexander Nast gefehlt.

Die Online-Version des deutschen Originalbeitrags ist zu finden unter: http://dx.doi.org/10.1007/ s15007-015-0929-1
Englische Fassung

http://link.springer. com/journal/40629

\section{Tab. 8: Ausgewählte Nahrungsmittelallergene und ihre Quellen pflanzlicher Herkunft $\mathrm{t}^{\mathrm{a}, \mathrm{b}}$}

\begin{tabular}{|c|c|c|c|c|c|c|c|c|}
\hline & \multicolumn{8}{|c|}{ Proteinfamilien } \\
\hline & \multirow[b]{3}{*}{$\begin{array}{l}\text { Bet-v-1- } \\
\text { Homologe }\end{array}$} & \multirow[b]{3}{*}{ LTP } & \multirow[b]{3}{*}{ Profiline } & \multirow[b]{3}{*}{ Thaumatine } & \multirow[b]{3}{*}{ Oleosine } & \multicolumn{3}{|c|}{ Speicherproteine (Proteinfamilien, Struktur) } \\
\hline & & & & & & \multirow{2}{*}{$\begin{array}{l}\text { Prolamine } \\
\text { 2S Albumine }\end{array}$} & \multicolumn{2}{|l|}{ Cupine } \\
\hline & & & & & & & $\begin{array}{l}\text { 7/8S-Globulin } \\
\text { (Vicilin) }\end{array}$ & $\begin{array}{l}\text { 11S-Globulin } \\
\text { (Legumin) }\end{array}$ \\
\hline Apfel & Mal d 1 & Mal d 3 & Mal d 4 & Mal d 2 & & & & \\
\hline Erdnuss & Ara h 8 & Ara h 9 & Arah 5 & & $\begin{array}{l}\text { Ara h } 10(16 \mathrm{kD}) \\
\text { Ara h } 11(14 \mathrm{kD})\end{array}$ & $\begin{array}{l}\text { Ara h } 2 \\
\text { Ara h } 6 \\
\text { Ara h } 7\end{array}$ & Ara h 1 & Arah 3 \\
\hline $\begin{array}{l}\text { Gewürze } \\
\text { Paprika } \\
\text { Petersilie }\end{array}$ & Pet c 1 & Pet c 3 & $\begin{array}{l}\text { Cap a } 2 \\
\text { Pet c } 2\end{array}$ & Сар а 1 & & & & \\
\hline Haselnuss & Cor a 1 & Cor a 8 & Cor a 2 & & $\begin{array}{l}\text { Cor a } 12(17 \mathrm{kD}) \\
\text { Cor a } 13 \\
(14 / 16 \mathrm{kD})\end{array}$ & Cor a 14 & Cor a 11 & Cor a 9 \\
\hline Karotte & Dau c 1 & Dau c 3 & Dau c 4 & & & & & \\
\hline Kirsche & Pru av 1 & Pru av 3 & Pru av 4 & Pru av 2 & & & & \\
\hline Kiwi & Act $d 8$ & & Act d 9 & Act d 2 & & & & \\
\hline Pfirsich & Prup 1 & Pru p 3 & Pru p 4 & & & & & \\
\hline Sellerie & Api $g 1$ & & Api g 4 & & & & & \\
\hline Sesam & & & & & $\begin{array}{l}\text { Ses i } 4 \\
\text { Ses i } 5\end{array}$ & $\begin{array}{l}\text { Ses i } 1 \\
\text { Ses i } 2\end{array}$ & Sesi 3 & $\begin{array}{l}\text { Ses i } 6 \\
\text { Ses i } 7\end{array}$ \\
\hline Sojabohne & Gly m 4 & Gly m 1 & Gly m 3 & & & & Gly m 5 & Gly m 6 \\
\hline Weizen & & Tri a 14 & Tri a 12 & & & $\begin{array}{l}\text { Tri a } 19 \\
\text { ( } \omega-5-\text {-Gliadin })\end{array}$ & & \\
\hline
\end{tabular}


Allergo J Int 2015; 24: 334

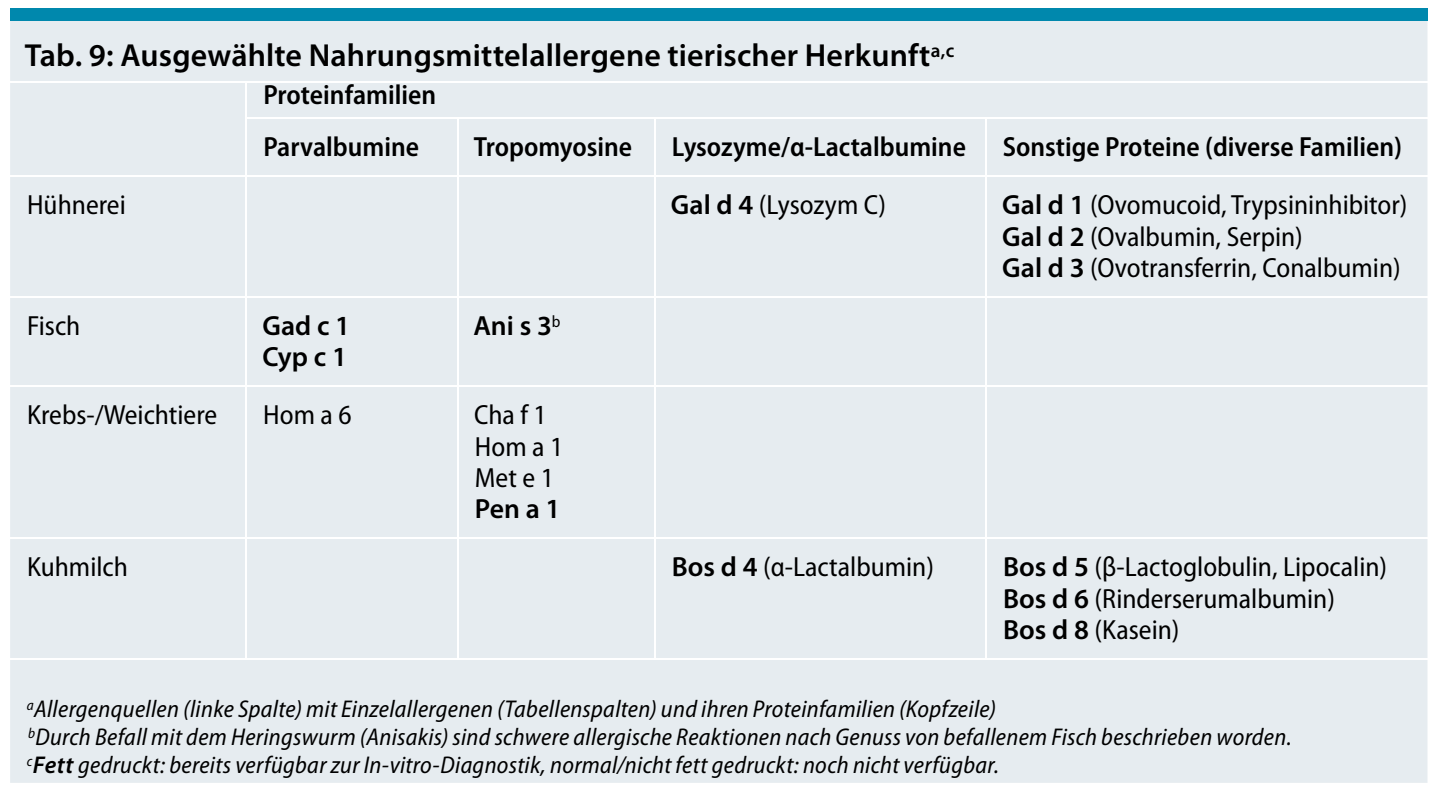

\section{Prof. Dr. Margitta Worm}

Allergie-Centrum-Charité

Klinik für Dermatologie, Venerologie und Allergologie

Charité - Universitätsmedizin Berlin

Charitéplatz 1

10117 Berlin, Deutschland

E-Mail: margitta.worm@charite.de

\section{Zitierweise}

Worm M, Reese I, Ballmer-Weber B, Beyer K, Bischoff SC, Claßen M, Fischer PJ, Fuchs T, Huttegger I, Jappe U, Klimek L, Koletzko B, Lange L, Lepp U, Mahler V, Nast A, Niggemann B, Rabe U, Raithel M, Saloga J, Schäfer C, Schnadt S, Schreiber J, Szépfalusi Z, Treudler R, Wagenmann M, Watzl B, Werfel T, Zuberbier T, Kleine-Tebbe J. Erratum to: Guidelines on the management of IgE-mediated food allergies. S2k-Guidelines of the German Society for Allergo- logy and Clinical Immunology (DGAKI) in collaboration with the German Medical Association of Allergologists (AeDA), the German Professional Association of Pediatricians (BVKJ), the German Allergy and Asthma Association (DAAB), German Dermatological Society (DDG), the German Society for Nutrition (DGE), the German Society for Gastroenterology, Digestive and Metabolic Diseases (DGVS), the German Society for Oto-Rhino-Laryngology, Head and Neck Surgery, the German Society for Pediatric and Adolescent Medicine (DGKJ), the German Society for Pediatric Allergology and Environmental Medicine (GPA), the German Society for Pneumology (DGP), the German Society for Pediatric Gastroenterology and Nutrition (GPGE), German Contact Allergy Group (DKG), the Austrian Society for Allergology and Immunology (ÖGAI), German Professional Association of Nutritional Sciences (VDOE) and the Association of the Scientific Medical Societies Germany (AWMF). Allergo J Int 2015;24:333-4

DOI: $10.1007 / \mathrm{s} 40629-015-0075-\mathrm{z}$ 